\title{
ANALYTICALLY UNIFORM SPACES AND SOME APPLICATIONS $\left({ }^{(}\right)$
}

\author{
BY \\ LEON EHRENPREIS
}

Introduction. Let $W$ be a suitable space of functions or distributions. Then we shall show that any $T \in W$ admits a representation

$$
T(x)=\int \exp (i x \cdot z) d \mu(x) / k(z)
$$

where $\mu$ is a bounded measure with support on the whole complex space, $k$ is a suitable function, and where the integral converges in a suitable sense. ( $\mu$ and $k$ are not unique.) For many spaces $W$, we can make more precise statements about the possibility of choosing the support of $\mu$ suitable. An application is made to the representation of a distribution as a sum of holomorphic functions. An example of this result is that, in one variable, any distribution of Schwartz is the sum of a function holomorphic in the upper half-plane and one holomorphic in the lower half-plane, these holomorphic functions having boundary values in a suitable sense. A second application is given to the problem of global approximation of a function or a distribution. Thirdly, we show that every entire function is the initial and final value (i.e. Cauchy value) for a solution of the heat equation. We also use our methods to solve some problems of division posed in [3].

In a sense, the results described above are unsatisfactory because there is an undue emphasis put on holomorphic functions whereas we should like to consider the class of holomorphic functions merely as an example of solutions of a system of partial differential equations. Thus we should like to represent any distribution of Schwartz on the real line in the form $f_{1}+f_{2}$ where $f_{1}$ is defined in the upper half-plane, $f_{2}$ is defined in the lower half-plane, and $D f_{j}=0$ in the appropriate half-plane, where $D$ is a suitable differential operator in two variables. In case $D$ has constant coefficients and is of the form

$$
D=\partial^{m} / \partial y^{m}+P_{1}(\partial / \partial x) \partial^{m-1} / \partial y^{m-1}+\cdots+P_{m}(\partial / \partial x)
$$

where the $P_{j}$ are constant coefficient differential operators, then even more can be proven: Given any $m$-tuple $\left(T_{j}(x)\right)$ of distributions on the real axis, we can find distributions $f_{1}$ in the upper half-plane and $f_{2}$ in the lower halfplane such that $D f_{j}=0$ and

$$
\lim _{y \rightarrow 0+} \partial^{i}\left[f_{1}(x, y)\right] / \partial y^{i}+\lim _{y \rightarrow 0-} \partial^{i}\left[f_{2}(x, y)\right] / \partial y^{j}=T_{j}(x)
$$

Received by the editors December 29, 1960.

(1) Work supported by ONR 432 JLP. 
for $j=0,1, \cdots, m-1$ where the limits exist in the sense of distributions.

Thus, though in general there is no existence theorem for the problem for a half-plane with arbitrary distributions as initial data, there is existence for this modified Cauchy problem. It would be of interest to give other "boundary conditions" to make $f_{1}$ and $f_{2}$ unique.

The above result can be extended to higher dimensions and to general constant coefficient systems. The details will be presented elsewhere.

1. General representation theorem. Let $R(C)$ denote real (complex) euclidean space of dimension $n$; by $x=\left(x_{1}, \cdots, x_{n}\right)\left[z=\left(z_{1}, \cdots, z_{n}\right)\right]$ we denote the coordinates on $R(C)$. Let $W$ be a reflexive space of functions or distributions on $R$; assume that $W$ contains all exponential functions and that the mapping $z \rightarrow \exp (z \cdot x)$ is an analytic map of $C \rightarrow W$; assume also that the exponentials are dense in $W$. Denote by $W^{\prime}$ the dual of $W$; for an $S \in W^{\prime}$ we can define the Fourier transform

$$
\hat{S}(z)=S \cdot \exp (i z \cdot x)
$$

for any $z \in C . S(z)$ is an entire function on $C$ and $S$ determines $S$ uniquely.

We now introduce a most important hypothesis which we call:

Analytically uniform. There exists a family $K$ of continuous positive functions $k$ on $C$ so that if $W^{\prime}$ denotes the space of Fourier transforms of $W^{\prime}$ with the topology of $\hat{W}^{\prime}$ defined to make the Fourier transform a topological isomorphism, then the sets

$$
N_{k}=\left\{F \in \hat{W}^{\prime}|| F(z) \mid \leqq k(z) \text { for all } z \in C\right\}
$$

form for $k \in K$ a fundamental system of neighborhoods of zero in $\hat{W}^{\prime}$. Moreover, for any $F \in \hat{W}^{\prime}$ and any $k \in K,|F(z)| / k(z) \rightarrow 0$ as $|z| \rightarrow \infty$. We call $K$ an analytic uniform structure for $W$.

It is shown in $[1 ; 2 ; 3 ; 4]$ that most of the important spaces of functions and distributions which were considered by Laurent-Schwartz are analytically uniform. In particular, the spaces $H$ of entire functions, $E$ of indefinitely differentiable functions, $D^{\prime}$ of distributions and $D_{r}^{\prime}$ of distributions of finite order are analytically uniform. (For the space $H, R$ must be replaced by $C$, that is the $x_{j}$ must be considered as complex variables. For these examples see $[1 ; 2 ; 3 ; 4]$.

Now, for $W$ analytically uniform, let us consider any $T \in \hat{W}$, the dual of $\hat{W}^{\prime}$. Then $T$ is bounded on some neighborhood of zero in $\hat{W}^{\prime}$. Thus there exists a $k \in K$ so that the conditions: $F \in \hat{W}^{\prime}$,

$$
\max _{z \in C}|F(z)| / k(z) \leqq 1
$$

imply $|T \cdot F| \leqq 1$. By the Hahn-Banach theorem, $T$ can be extended to a continuous linear function $\tilde{T}$ on the space $L_{k}$ of continuous functions $G$ on $C$ such that $|G(z)| / k(z)$ is (for this $k$ ) bounded on $C$ and zero at infinity 
with the norm $\max |G(z) / k(z)|$. Hence there exists a measure $\mu$ on $C$ with total variation finite such that for any such $G$ we have

$$
\widetilde{T} \cdot G=\int G(z) d \mu(z) / k(z) .
$$

I want to use this representation to define the Fourier transform on $W$. First we notice that for any $S \in W^{\prime}$ the integral

$$
\int S \cdot \exp (i x \cdot z) d \mu(z) / k(z)=\int S(z) d \mu(z) / k(z)
$$

certainly exists in the sense of Lebesgue because of our assumptions on the topology of $W^{\prime}$. Moreover, these integrals are uniformly bounded for $S$ in a neighborhood of zero defined by $|\hat{S}(z)| / k(z) \leqq 1$ for $z \in C$. Thus, by the reflexivity of $W$, the map

$$
S \rightarrow \int S(z) d \mu(z) / k(z)
$$

defines an element of $W$ which we call the Fourier transform of $T$ and we denote it by $\hat{T}$. Symbolically we write

$$
\hat{T}(x)=\int \exp (i x \cdot z) d \mu(z) / k(z) .
$$

Note that in case $k$ is large at infinity, $\hat{T}$ will be a function.

I am not yet finished, for I want to show that every element of $W$ is of the form $\hat{T}$ for some $\hat{T} \in \hat{W}$. Let $U$ be any element of $W$. Then it is clear that the function

$$
\hat{S} \rightarrow S \cdot U, \quad \hat{S} \in \hat{W}^{\prime}
$$

represents an element $V \in \hat{W}$. Moreover we see immediately from the definitions that $U=\hat{V}$.

Finally it is clear that the correspondence $T \leftrightarrow \hat{T}$ is one-one between $\hat{W}$ and $W$. (Of course, there are many measures $\mu$ which represent $T$.) Thus we have proven

THEOREM 1. For any $T \in \hat{W}$ we can find a bounded measure $\mu$ on $C$ and $a$ $k \in K$ so that

$$
T \cdot \hat{S}=\int \hat{S}(z) d \mu(z) / k(z)
$$

for any $\hat{S} \in \hat{W}^{\prime}$ where the integral exists in the sense of Lebesgue-Stieltjes. If we set 


$$
\hat{T} \cdot S=T \cdot \hat{S}
$$

for $S \in \hat{W}^{\prime}$ then the map $T \rightarrow \hat{T}$ is a one-one linear map of $\hat{W}$ onto $W$. Thus every element of $W$ can be represented by a symbolic integral

$$
\int \exp (i x \cdot z) d \mu(z) / k(z)
$$

for some $k \in K$ and some bounded measure $\mu$.

For many important function spaces the symbolic integral will actually exist as a Lebesgue integral. This is in particular true of the spaces $E$ and $H$.

2. Strengthened representation theorem. As we mentioned above, the measure $\mu$ of Theorem 1 is not unique. In many cases we can say much more. For example, if $T$ satisfies a partial differential equation then the support of $\mu$ can be chosen to be an algebraic variety. This result is very deep and is explained in [5]. We content ourselves here with showing that in certain cases the support of $\mu$ can be chosen to be on certain subsets of $C$.

We explain how these results are derived: First let us note that the measure $\mu$ was found because of the representation of the elements of the dual of $L_{k}$ as bounded measures on $C$ divided by $k$. Now, let $s$ be a closed subset of $C$ and denote by $L_{k}(s)$ the space of functions $G$ which are continuous on $s$, satisfy $|G(z)| / k(z)$ is bounded on $s$, and tends to zero as $|z| \rightarrow \infty$; the norm in $L_{k}(s)$ is defined as $\max _{z \in z}|G(z)| / k(z)$.

It is easy for many $k$ to produce sets $s$ so that the conditions $G \in \hat{W}^{\prime}$ and

$$
\max _{z \in \ell}|G(z)| / k(z) \leqq 1
$$

imply

$$
\max _{z \in C}|G(z)| / k(z) \leqq 1 .
$$

Even if this cannot be done, then we can usually produce an $s$ and a $k_{1} \in K$ so that the conditions $G \in \hat{W}^{\prime}$,

$$
\max _{z \in \delta}|G(z)| / k_{1}(z) \leqq 1
$$

imply

$$
\max _{z \in C}|G(z)| / k(z) \leqq 1 .
$$

If this can be done then we say that $\left(s, k_{1}\right)$ is $k$ sufficient.

Suppose that $\left(s, k_{1}\right)$ is $k$ sufficient. Then if $T \in \hat{W}$ is continuous on $\hat{W}^{\prime}$ in the topology of $L_{k}$, then $T$ is also continuous on $\hat{W}^{\prime}$ in the topology induced by $L_{k_{1}}(s)$. The method of $\S 1$ then leads to the representation 


$$
\hat{T}(x)=\int \exp (i x \cdot z) d \mu(z) / k_{1}(z)
$$

where $\mu$ is a bounded measure with support $\mu \subset s$. In particular we have

Lemma 2. Suppose there exists a closed set $s \subset C$ so that for each $k$ in a subset $K_{1}$ of $K$, this subset being sufficient to define the topology of $W^{\prime}$, we can find a $k_{1}$ in $K$ so that $\left(s, k_{1}\right)$ is $k$ sufficient; then every $\hat{T} \in W$ has the Fourier representation

$$
\hat{T}(x)=\int \exp (i x \cdot z) d \mu(z) / k_{1}(z)
$$

for some $k_{1} \in K$ where $\mu$ is a bounded measure with support on s.

Examples of sets $s$ are the complex plane minus any compact set, as follows from the maximum modulus theorem. Using the Phragmén-Lindelöf method we can make the sets $s$ even smaller. We illustrate this below in several examples.

The space $H . H$ is the space of entire functions of $x$. (The $x_{j}$ are considered here as complex variables.) $\hat{H}^{\prime}$ is the space of entire functions of exponential type on $C$. $H$ is analytically uniform and the set $K$ consists of all functions $k$ which are continuous, positive, and such that

$$
\exp (A|z|)=O(k(z))
$$

for any $A>0$ (see [2]).

LEMмa 3. Sets $s$ satisfying the conditions of Lemma 2 can be constructed as follows: For each $j$ let $A_{j}$ and $B_{j}$ be any two distinct straight lines through the origin in the complex $z_{j}$ plane. Then we can choose $s$ as the cartesian product $\left(A_{1} \cup B_{1}\right) \times \cdots \times\left(A_{n} \cup B_{n}\right)$.

Proof. For simplicity, we consider first the case $n=1$; the passage to $n>1$ will be discussed below. We write $A$ for $A_{1}$ and $B$ for $B_{1}$. We may clearly assume that $A$ is the real axis; let $\alpha$ be the counterclockwise angle between the positive real axis and $B$.

We map the sector of angle $\alpha$ onto the upper half-plane by means of the mapping $z \rightarrow z^{\pi / \alpha}=\lambda$ say. For any $k \in K$, we have $k(|z|)=k(|\lambda| \alpha / \pi)$ so $k$ is larger at infinity than $\exp (t|\lambda| \alpha / \pi)$ for any $t>0$. We need

LEMMA 4. Let $M$ be a continuous function defined on $R$ such that $M(x) \geqq 1$ and $M(x) \geqq|x|^{\alpha / x}$ for all $x$ and such that, at infinity, $M(\lambda)$ is larger than $t|\lambda|^{\alpha / \pi}$ for any $t>0$. Then there is a positive monotonic function $\tilde{M}$ on $R$ which is real analytic except for a discrete sequence of jumps, with $\tilde{M}$ larger than $t|\lambda|^{\alpha / \pi}$ at infinity for each $t>0$, and satisfying

$$
\begin{aligned}
\tilde{M}(x) & \leqq M(x), \\
\tilde{M}(x+y) & \leqq 4 \tilde{M}(x)+4 \tilde{M}(y),
\end{aligned}
$$




$$
\begin{aligned}
\tilde{M}(x y) & \leqq 2 \tilde{M}(x) \tilde{M}(y), \\
\int_{-\infty}^{\infty} \frac{\tilde{M}(x) d x}{1+x^{2}} & <\infty .
\end{aligned}
$$

Before proving Lemma 4, we shall use it to complete the proof of Lemma 3. We construct the Poisson integral $M^{*}$ of $\tilde{M}$ for the upper half-plane:

$$
M^{*}(x, y)=\frac{1}{\pi} \int_{-\infty}^{\infty} \frac{y \tilde{M}\left(x^{\prime}\right)}{\left(x-x^{\prime}\right)^{2}+y^{2}} d x^{\prime}
$$

We shall show first that $M^{*}(x, y) \leqq$ const $[\tilde{M}(x)+\tilde{M}(y)]$. For this purpose we write

$$
M^{*}=\int_{-\infty}^{x}+\int_{x}^{\infty}
$$

Now,

$$
\begin{aligned}
\int_{-\infty}^{x} \frac{y \tilde{M}\left(x^{\prime}\right)}{\left(x-x^{\prime}\right)^{2}+y^{2}} d x^{\prime} & =\int_{-\infty}^{0} \frac{y \tilde{M}\left(x^{\prime}+x\right)}{x^{\prime 2}+y^{2}} d x^{\prime} \\
& \leqq 4 \int_{-\infty}^{0} \frac{y\left[\tilde{M}\left(x^{\prime}\right)+\tilde{M}(x)\right]}{x^{\prime 2}+y^{2}} d x^{\prime} \\
& =4 \tilde{M}(x) \int_{-\infty}^{0} \frac{y}{x^{\prime 2}+y^{2}} d x^{\prime}+4 \int_{-\infty}^{0} \frac{y M\left(x^{\prime}\right)}{x^{\prime 2}+y^{2}} d x^{\prime}
\end{aligned}
$$

The first term is just $2 \pi \tilde{M}(x)$. The second is

$$
\begin{aligned}
4 \int_{-\infty}^{0} \frac{\tilde{M}\left(x^{\prime}\right) d x^{\prime} / y}{1+\left(x^{\prime} / y\right)^{2}} & \leqq \text { const } \int_{-\infty}^{0} \frac{\tilde{M}\left(x^{\prime} / y\right) \tilde{M}(y) d x^{\prime} / y}{1+\left(x^{\prime} / y\right)^{2}} \\
& =\operatorname{const} \tilde{M}(y) \int_{-\infty}^{0} \frac{\tilde{M}\left(x^{\prime} / y\right) d x^{\prime} / y}{1+\left(x^{\prime} / y\right)^{2}} \\
& =\operatorname{const} \tilde{M}(y) .
\end{aligned}
$$

On the other hand we have

$$
\int_{x}^{\infty} \frac{y \tilde{M}\left(x^{\prime}\right)}{\left(x^{\prime}-x\right)^{2}+y^{2}} d x^{\prime}=\int_{0}^{\infty} \frac{y \tilde{M}\left(x^{\prime}+x\right)}{x^{\prime 2}+y^{2}} d x^{\prime},
$$

which is handled as above. Putting the above calculations together we deduce

$$
M^{*}(x, y) \leqq \operatorname{const}[\tilde{M}(x)+\tilde{M}(y)],
$$

which is the desired result.

In particular we derive the fact that 


$$
M^{*}(x, y) \leqq \text { const } \tilde{M}(|\lambda|)
$$

where $\lambda=x+i y$.

Next, we want to show that $M^{*}$ is large at infinity; in fact, we want to show that, at infinity, $M^{*}(x, y)>t|\lambda| \alpha / \pi$ for any $t>0$. For this we use the fact that $\tilde{M}$ is positive and monotonic. Then for $x \geqq 0$,

$$
M^{*}(x, y) \geqq \int_{0}^{\infty} \frac{y \tilde{M}\left(x+x^{\prime}\right)}{x^{\prime 2}+y^{2}} d x^{\prime} .
$$

For any $t$ there is a $t^{\prime}\left(\right.$ possibly $\left.t^{\prime}<0\right)$ so that

$$
\tilde{M}\left(x+x^{\prime}\right) \geqq t^{\prime}+t\left(x+x^{\prime}\right)^{\alpha / \pi} .
$$

Thus,

$$
\begin{aligned}
M^{*}(x, y) & \geqq \frac{\pi}{2} t^{\prime}+t \int_{0}^{\infty} \frac{y\left(x+x^{\prime}\right)^{\alpha / \pi}}{x^{\prime 2}+y^{2}} d x^{\prime} \\
& \geqq \frac{\pi}{2} t^{\prime}+t \int_{0}^{\infty} \frac{y\left(x^{\alpha / \pi}+x^{\prime \alpha / \pi}\right)}{x^{\prime 2}+y^{\prime 2}} d x^{\prime} \\
& =\frac{\pi}{2} t^{\prime}+\frac{\pi}{2} t x^{\alpha / \pi}+\frac{\pi}{2} t y^{\alpha / \pi}
\end{aligned}
$$

Since $x, y \geqq 0$ this means that, at infinity,

$$
\begin{aligned}
M^{*}(x, y) & \geqq \frac{\pi}{2} t\left(x^{\alpha / \pi}+y^{\alpha / \pi}\right) \\
& \geqq c t|\lambda|^{\alpha / \pi}
\end{aligned}
$$

where $c$ is some constant independent of $t$. This gives our desired result for $x \geqq 0$ and the case $x<0$ is similar.

We wish now to complete the proof of Lemma 3 in case $n=1$. It is easily seen that we may assume the functions $k \in K$ are functions of $|z|$. Let $k \in K$ be given; it is sufficient to show that we can find, for each of the four sectors $\Lambda_{j}$ into which the lines $A$ and $B$ divide $C$, a $k_{j} \in K$ (or rather, a $k_{j}$ which is positive and tends to infinity faster than any exponential (but $k_{j}$ may not be continuous)) so that the conditions $F \in \hat{H}^{\prime}$,

$$
|F(z)| / k_{j}(z) \leqq 1 \text { for } z \text { on boundary } \Lambda_{j}
$$

imply

$$
|F(z)| / k(z) \leqq \text { const for } z \in \Lambda_{j}
$$

For simplicity of notation we restrict ourselves to $\Lambda_{1}$ which is the sector formed by: 


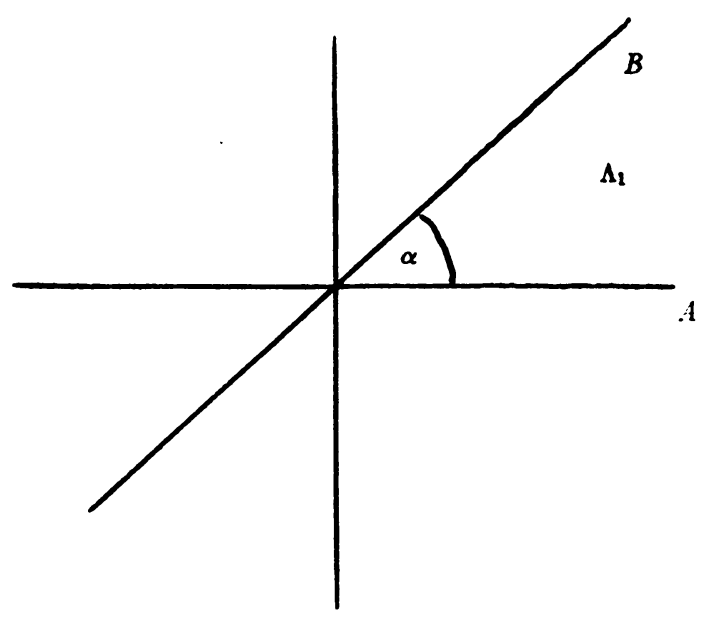

We use the notation of Lemma 4: Set $M(\lambda)=(1 / 2 c) \log k(\lambda)$, where $c$ is the constant occurring in (17) and then define $k_{1}(z)=\exp (\tilde{M}(z))$. Let $M^{* *}(\lambda)$ be a conjugate of $M^{*}(\lambda)$ and set

$$
k_{1}(z)=\exp \left[M^{*}(z)+i M^{* *}(z)\right]
$$

for $z \in \Lambda_{1}$.

From (19) we deduce that on the boundary of $\Lambda_{1}$ we have $\left|k_{1}(z)\right|>\exp (t|z|)$ at infinity for any $t>0$.

Now, suppose $F \in \hat{H}^{\prime}$ and $|F(z)| / k_{1}(z) \leqq 1$ for $z \in$ boundary $\Lambda_{1}$. Then, $\left|k_{1}(z)\right|=k_{1}(z)$ for $z \in$ boundary $\Lambda_{1}$ except at the discontinuities of $\tilde{M}$, but these points do not matter because $F$ and $k$ are continuous everywhere, so we can neglect them. [It is also possible to modify $\tilde{M}$ on the sets $x_{j}-1 / 2 \leqq x$ $\leqq x_{j}+1 / 2$ (see the construction below) to make $\widetilde{M}$ indefinitely differentiable by changing slightly the constants that occur in the conditions on $\tilde{M}$. We have thus $\left|F(z) / k_{1}(z)\right| \leqq 1$ for $z \in$ boundary $\Lambda_{1}$.] The function $F(z) / k_{1}(z)$ is analytic in $\Lambda_{1}$; by (19) and the fact that $F$ is of exponential type it follows that $\left|F(z) / k_{1}(z)\right| \rightarrow 0$ as $|z| \rightarrow \infty, z \in \Lambda_{1}$. Thus by the maximum modulus principle, $\left|F(z) / k_{1}(z)\right| \leqq 1$ for $z \in \Lambda_{1}$. That is, for $z \in \Lambda_{1}$ we have

$$
\begin{aligned}
|F(z)| & \leqq\left|k_{1}(z)\right| \\
& =\exp \left(M^{*}(z)\right) \\
& \leqq \exp (c \tilde{M}(|z|)) \\
& \leqq \exp (c(M(\lambda)) \\
& =k(|z|) .
\end{aligned}
$$

This is the desired result.

Passage to $n>1$. There is no essential difficulty involved. We notice that 
the functions $k \in K$ could be chosen to be products $k(z)=k^{1}\left(z_{1}\right), \cdots, k^{n}\left(z_{n}\right)$. In each of the complex $z_{j}$ planes we have four sectors $\Lambda_{l}^{j}, k=1,2,3,4$. Then given any cartesian product of sectors $\prod \Lambda_{i}^{j}$ we must produce a $k_{1} \in K$ such that the conditions $F \in \hat{A}^{\prime}$,

$$
|F(z)| / k_{1}(z) \leqq 1, \quad z \in \text { distinguished boundary of } \prod \Lambda_{l_{j}}^{j},
$$

imply

$$
|F(z)| / k(z) \leqq \text { const, } \quad z \in \Pi \Lambda_{i_{j}}^{j} .
$$

We construct $k_{1}$ as a product $k_{1}(z)=k_{1}^{1}\left(z_{1}\right) \cdots k_{1}^{n}\left(z_{n}\right)$ where $k_{1}^{j}$ is constructed for $k^{i}$ and $\Lambda_{i_{j}}^{j}$ by our above one dimensional argument. The corresponding functions $k^{j *}$ and $k^{j}$ are constructed as above. The one dimensional maximum modulus principle is replaced by the fact that a function which is holomorphic in $\Pi \Lambda_{i_{j}}^{j}$ and tends to zero at infinity will take its maximum on the distinguished boundary. This concludes the passage to $n>1$.

Proof of Lemma 4. The proof of Lemma 3 will be complete when we complete the proof of Lemma 4, that is the construction of $\tilde{M}$ with the desired properties. Since $\tilde{M}$ is even, we construct $\tilde{M}(x)$ for $x>0$. For simplicity we assume $\tilde{M}(x)$ as equal to const $x^{\alpha / \pi}$ for long intervals. More precisely, we shall construct a sequence $\left\{x_{j}\right\}$ with $x_{j+1}>x_{j}>0$ and we define

$$
\tilde{M}(x)=\left\{\begin{array}{lr}
1 & \text { for } 0 \leqq x<x_{1} \\
j x^{\alpha / \pi} & \text { for } x_{j} \leqq x<x_{j+1} .
\end{array}\right.
$$

We must construct $\left\{x_{j}\right\}$ so that $\tilde{M}$ will have all the desired properties.

It is clear that $\tilde{M}$ is positive, monotonic, even, and $\tilde{M}$ is larger at infinity than any $t x^{\alpha / r}$. Next we note that for any $\alpha$

$$
\begin{aligned}
(x+y)^{\alpha / \pi} & \leqq 2^{\alpha / \pi} \max \left(x^{\alpha / \pi}, y^{\alpha / \pi}\right) \\
& \leqq 2 x^{\alpha / \pi}+2 y^{\alpha / \pi} .
\end{aligned}
$$

We choose $x_{1}=1$ and we choose the $x_{j}$ so lacunary that $\tilde{M}(x) \leqq M(x)$, and $x_{j+1}>2 \sum_{l_{\leq j}} x_{j}$ and $x_{j+1}>\left(x_{j}\right)^{2}$. Moreover we assume that $j x_{j}^{a / x-1}<j^{-2}$. (Such a choice is certainly possible since $\alpha<\pi$.) Then we have for $\tilde{M}(x+y)$ the following inequality: Suppose $x \geqq y$; then if $j_{0}$ is the smallest $j$ such that $x<x_{j_{0}}$, then $x+y<x_{j_{0}+1}$. Thus, if $j_{0} \geqq 2$ then

$$
\tilde{M}(x)=j_{0} x^{\alpha / \pi}
$$

and

$$
\begin{aligned}
\tilde{M}(x+y) & \leqq\left(j_{0}+1\right)(x+y)^{\alpha / x} \\
& \leqq 2^{\alpha / x}\left(j_{0}+1\right) \tilde{M}(x) / j_{0} \\
& \leqq 4 \tilde{M}(x) \\
& \leqq 4 \tilde{M}(x)+4 \tilde{M}(y) .
\end{aligned}
$$


If $j_{0}=1$ then $\tilde{M}(x+y) \leqq 2^{\alpha / x}<2<4 \tilde{M}(x)+4 \tilde{M}(y)$. Thus, condition (12) is satisfied.

To verify condition (13), suppose $x, y>1$. Suppose again that $x \geqq y$ and that $j_{0}$ is the smallest $j$ so that $x<x_{j_{0}}$. Then $x y<x^{2}<x_{j_{0}}^{2}<x_{j_{0}+1}$. Thus, $\tilde{M}(x y)$ $\leqq\left(j_{0}+1\right) x^{\alpha / \pi}$ while $\tilde{M}(x)=j_{0} x^{\alpha / \pi}$. This means that

$$
\begin{aligned}
\tilde{M}(x y) & \leqq 2 \tilde{M}(x) \\
& \leqq 2 \tilde{M}(x) \tilde{M}(y) .
\end{aligned}
$$

In case either $x$ or $y$ is $\leqq 1$, say $y \leqq 1$, then $x y \leqq x$, so $\tilde{M}(x y) \leqq \widetilde{M}(x)=M(x) M(y)$. Thus condition (13) is verified.

Finally, for condition (14) we note that for $j \geqq 1$,

$$
\begin{aligned}
\int_{x}^{x_{j+1}} \frac{\tilde{M}(x)}{1+x^{2}} d x & =j \int_{x_{j}}^{x_{j+1}} \frac{x^{\alpha / \pi}}{1+x^{2}} d x \\
& \leqq j \int_{x_{j}}^{x_{j+1}} x^{\alpha / \pi-2} d x \\
& =\frac{j}{\alpha / \pi-1}\left[x_{j+1}^{\alpha / \pi-1}-x_{j}^{\alpha / \pi-1}\right] \\
& <\text { const } / j^{2}
\end{aligned}
$$

by construction of the $x_{j}$. Thus,

$$
\sum_{j=1}^{\infty} \int_{x_{j}}^{x_{j+1}} \frac{\tilde{M}(x)}{1+x^{2}} d x<\infty
$$

which is the desired result. $\left({ }^{2}\right)$

From Lemmas 2 and 3 and the statements about the topology of $\hat{H}^{\prime}$ we deduce (the last two statements of Theorem 5 are obvious):

TheOREM 5. Let $A_{j}, B_{j}$ be as in the statement of Lemma 3. Then for any entire function $f$ we can find a function $k$ on $C$ which tends to zero faster than any exponential and we can find a bounded measure $\mu$ with support $\mu \subset \prod\left(A_{j} \cup B_{j}\right)$ such that

$$
f(x)=\int \exp (i x \cdot z) d \mu(z) / k(z) .
$$

The integral converges absolutely and uniformly for $x$ in any compact set of the complex space. Conversely, any such integral represents an entire function.

(2) Added in proof. Professor L. Rubel has pointed out that the proof of Lemma 3 can be greatly simplified by replacing the function $\tilde{k}_{1}(z)$ by a series of the form $\sum a_{j} \exp (j z)$ where the $a_{f}$ are suitably chosen. The method of Rubel is valid for many other spaces, but does not seem to have as wide applicability as the method used in the proof of Lemma 4. 
REMARK. We could get an even more precise result by replacing the sets $A_{j} \cup B_{j}$ by any three half-lines through the origin such that each of the sectors formed is of opening $<\pi$. The proof of this is the same as the above proof.

We should like to reformulate Theorem 5 in classical language. For this purpose we restrict ourselves to the case $n=1$, and we choose $A=A_{1}$ as the real axis and $B=B_{1}$ as the imaginary axis. Then any entire function $f$ can be represented in the form

(27) $f(x)=\int \exp (i x z) d \mu_{1}(z) / k_{1}(z)+\int \operatorname{expix} z d \mu_{2}(z) / k_{2}(z)=f_{1}+f_{2}$, say,

where $k_{1}$ and $k_{2}$ are continuous, positive, monotonic functions which grow faster than any exponential and $\mu_{1}$ and $\mu_{2}$ are bounded measures with support $\mu_{1} \subset A$ and support $\mu_{2} \subset B$.

We want to investigate $f_{1}$ and $f_{2}$. It is easily seen that (writing $x=\xi+i \eta$ )

$$
\left|f_{1}(\xi+i \eta)\right| \leqq \int \exp (|\eta z|)\left|d \mu_{1}(z)\right| / k_{1}(z)=g(\eta), \text { say. }
$$

This means that $f_{1}$ is uniformly bounded in strips parallel to the real axis. Similarly, $f_{2}$ is uniformly bounded in strips parallel to the imaginary axis. I do not know if one can give a direct proof of such a representation of an arbitrary entire function.

It should be noted that the above is not quite a characterization of the functions $f_{j}$. However, it is "almost" a characterization, for if $f_{1}$ were $L_{2}$ on lines parallel to the real axis and $f_{2}$ were $L_{2}$ on lines parallel to the imaginary axis then we would have a representation

$$
f_{j}(x)=\int \exp (i x z) d \mu_{j}(z) / k(z) .
$$

Results for other spaces. We now give the result for the space $E$ which is analogous to Theorem 5 for the space $H$.

Theorem 6. For each $j=1,2, \cdots, n$, let $A_{j}$ denote the real axis in the complex $z_{j}$ plane and let $B_{j}$ be any other line through the origin. For any indefinitely differentiable function $f$ we can find continuous positive functions (of one complex variable) $l_{1}^{j}$ and $l_{2}^{j}$ with $l_{1}^{y}$ tending to infinity faster than any exponential and $l_{2}^{j}$ tending to infinity faster than any polynomial and we can find a bounded measure $\mu$ with support $\mu \subset \prod\left(A_{j} \cup B_{j}\right)$ such that

$$
f(x)=\int \exp (i x \cdot z) d \mu(z) / l(z)
$$

where, for $z=\left(z_{1}, \cdots, z_{n}\right)$, we have $l(z)=\prod l_{p}^{j}\left(z_{j}\right)$, where $p=1$ if $z_{j} \in B_{j}$ and $p=2$ if $z_{j} \in A_{j}$. 
The integral and each of its derivatives converge uniformly for $x$ in any compact set of $R$. Conversely, every such integral represents an $f \in E$.

The proof of Theorem 6 follows along essentially the same lines as that of Theorem 5 . We use the fact that $E$ is analytically uniform, the set $K$ consisting of all functions $k(z)$ which grow faster at infinity than any polynomial in $z$ times any $\exp (t|I z|)$ where $I z$ represents the imaginary part of $z$. (For this see [2].)

A result similar to Theorem 6 holds for the space $D_{r}^{\prime}$ of distributions of finite order. The only difference is that the functions $l_{2}^{\prime}$ satisfy $\left(1+\left|x_{j}\right|\right)^{-p}$ $=O\left(l_{2}^{\prime}\left(x_{j}\right)\right)$ for some $p>0$.

The space $D^{\prime}$ of distributions is also analytically uniform (see [3]). The set $K$ consists of all positive, continuous functions $k(z)$ on $C$ such that if $h(z)$ is any continuous function on $C$ such that for some $t>0$ we have

$$
h(z)=O\left(\exp (t|T z|) /(1+|z|)^{p}\right)
$$

for all $p$ then also

$$
h(z)=O(k(z)) .
$$

Let us notice the following:

LEMмa 7. Let $\omega(y)$ be any positive, continuous, monotonic function of the real variable $y$ such that, at infinity, $\log (y) / \omega(y) \rightarrow 0$. Let $R_{\omega}$ be the set in $C$ defined by

$$
|I z| \leqq \omega|(R z)|) \text {. }
$$

Then outside of $R_{\omega}$ "the topologies of $\hat{D}$ and $\hat{E}^{\prime}$ are the same," that is, given any $k$ of the set $K$ defining the analytically uniform structure for $D$ there is a $k$ of the set defining the analytically uniform structure for $\hat{E}^{\prime}$ such that $k=\hat{k}$ outside of $R_{\omega}$.

Proof. We give the proof for $n=1$; there are no difficulties involved in the extension to $n>1$. We define $\hat{k}$ as in the diagram on the following page. On the line joining $z$ with $z^{\prime}$ we define $\hat{k}$ by linear interpolation. We wish to verify that $\hat{k}(z)$ satisfies

$$
\exp (t|I z|)(1+|z|)^{p}=O(\hat{k}(z))
$$

so $k$ satisfies the desired properties.

Before proving this, let us explain why it should be so. On $|I z|=\omega(|z|)$ we have

$$
\exp (t|I z|)=\exp (t \omega(|R z|))
$$

Since $\omega(y) / \log (y) \rightarrow \infty$, the function $\exp (t \omega(|R z|))$ is larger at infinity than any polynomial. Since, in general, the only difference between the functions 


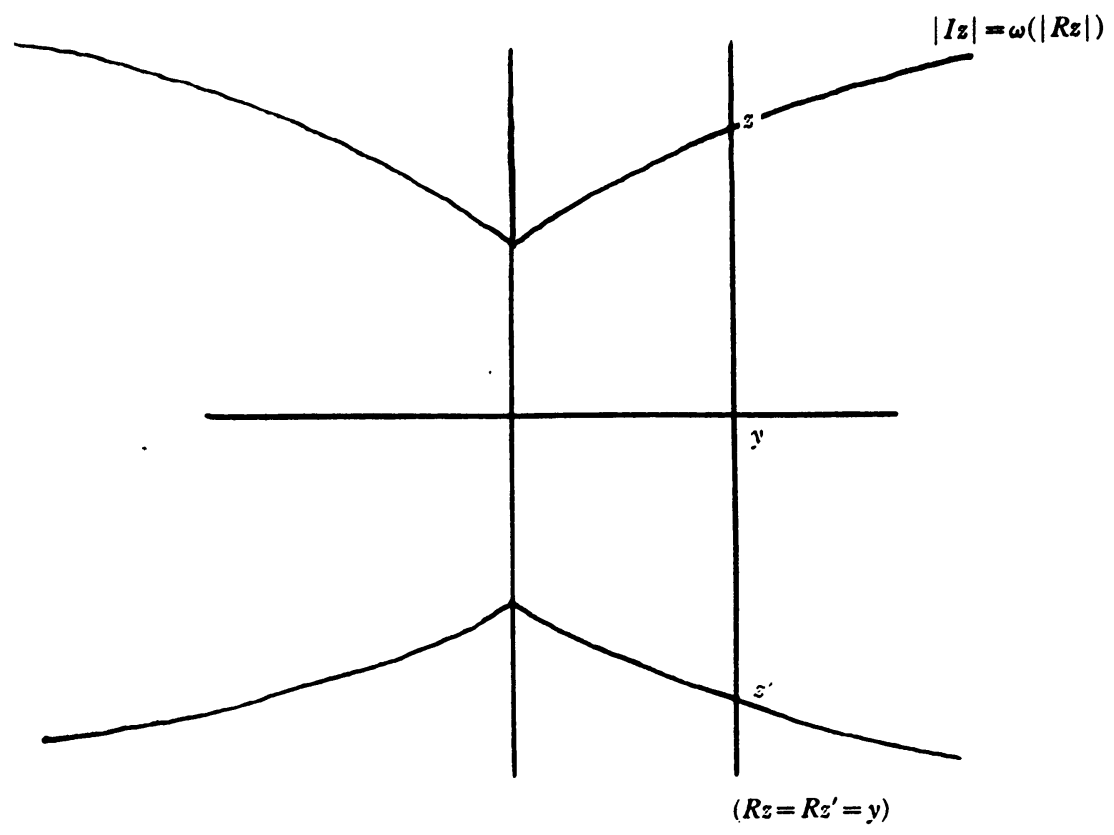

defining the uniform structures for $\hat{E}^{\prime}$ or $\hat{D}$ is a question of polynomial growth, we should expect our result.

To prove (32), it is sufficient to prove (32) outside $R_{\omega}$, as is easily seen. For simplicity we assume that $\omega(y)>2$ for all $y$. Then for any $p, t$ let

$$
f_{p, t}(z)= \begin{cases}0 & \text { for }|I z|<\omega(|R z|)-1 \\ \exp (t|I z|)(1+|z|)^{p} & \text { for } z \notin R_{\omega} .\end{cases}
$$

The definition of $f_{p, t}$ is completed by requiring that $f_{p, t}$ be continuous and $f_{p, t}$ is monotonically decreasing on the line joining $z$ with $y$ or $z^{\prime}$ with $y$ (see diagram).

I claim that for any $q$ we have

$$
f_{p, t}(z)=O\left(\exp ((t+1)|I z|) /(1+|z|)^{q}\right) .
$$

Now (34) is certainly satisfied if $|I z|<\omega(|R z|)-1$ for then the left side is 0 . For $I z \geqq \omega(|R z|)-1$ we need only prove that for any $q$

$$
(1+|z|)^{p+q}=O(\exp (|I z|))
$$

that is,

$$
\log (1+|z|) \leqq \text { const }+\frac{|I z|}{p+q}
$$

or, what is the same thing, 


$$
\log (1+|z|) /|I z| \rightarrow 0
$$

as $|z| \rightarrow \infty,|I z| \geqq \omega(|R z|)-1$. If (35) were violated then there would exist a sequence of points $z_{j}$ such that

$$
\left|I z_{j}\right| \geqq \omega\left(\left|R z_{j}\right|\right)-1
$$

and

$$
\log \left(1+\left|z_{j}\right|\right) /\left|I z_{j}\right| \geqq 1 \text { say. }
$$

But for $\left|z_{j}\right|$ large, $\log \left(1+\left|z_{j}\right|\right)>\left|I z_{j}\right|$ implies $\log \left|R z_{j}\right|>(1 / 2)\left|I z_{j}\right|$ which is impossible if $\left|I z_{j}\right| \geqq \omega(|R z|)-1$. This completes the proof of Lemma 7 .

REMARK. Lemma 7 is the key to the understanding of the nature of $C^{\infty}$ ellipticity (see $[3 ; 5]$ ).

THEOREM 8. Let $\omega$ satisfy the hypothesis of Lemma 7, and let $S$ be a distribution. Then there exists a measure $\mu$ with support $\mu \subset R_{\omega}$ and a function $k$ of the type used to define the analytic uniform structure of $D^{\prime}$ so that, if

$$
T=\int \exp (i x \cdot z) d \mu(z) / k(z)
$$

(symbolic integral) then $S-T \in E$.

Proof. Let $K$ denote the set of functions defining the analytic uniform structure for $D^{\prime}$ and $K^{\prime}$ the corresponding set for $E$. Then Lemma 7 shows that the topology of $\hat{D}$ is defined by the semi-norms

$$
\max _{z \in C}|F(z)| / k^{\prime}(z), \quad \quad k^{\prime} \in K^{\prime},
$$

and

$$
\max _{z \in R_{\omega}}|F(z)| / k(z), \quad k \in K,
$$

for $F \in D$. The method of proof of Theorem 1 then shows that we can write $S$ in the form

$$
S=\int \exp (i x \cdot z) d \mu(z) / k(z)+\int \exp (i x \cdot z) d \mu^{\prime}(z) / k^{\prime}(z)
$$

where $k \in K, k^{\prime} \in K^{\prime}$, and support $\mu \subset R_{\omega}$. The second integral on the right of (38) is certainly in $E$ which is the desired result.

The conditions imposed on $\omega$ in Lemma 7 are sufficient to obtain the conclusion of Theorem 8 for all distributions $S$. However, for particular distributions $S$ we should be able to obtain a representation for $T$ as in (37) where the support of $\mu$ is a smaller set. An example of the type of result we have in mind is the following: Suppose there exists a $k>0$ so that $S$ is a distribution of order $\leqq l(1+|x|)$ in the ball center origin, radius $|x|$. Then we 
can find a bounded measure $\mu$ whose support is contained in the set

$$
|I z| \leqq 1+l \log (1+|R z|)
$$

and a function $k$ of the type used to define the analytic uniform structure of $D^{\prime}$ such that for

$$
T=\int \exp (i x \cdot z) d \mu(z) / k(z)
$$

we have $S-T \in E$.

This result (which we have not proven) and similar results should be deducible by the methods used in [2] to describe the topology of $\hat{D}$. Hörmander and Malgrange have simplified the author's proof (their method is not yet published) and their method should shed more light on the situation.

3. Applications. It is well known and readily verified that every continuous function which is small at infinity $(n=1)$ can be represented as the sum of a function analytic in the upper half-plane with one analytic in the lower halfplane. We want to present a general treatment of this problem.

Let us denote by $C^{i}\left(j=1,2, \cdots, 2^{n}\right)$ the $2^{n}$ "octants" in $C$, that is, the closed subspaces defined by letting the imaginary parts have constant sign, real parts arbitrary; we set $C^{1}$ as the principal octant: $I z \geqq 0$ for all $l$. If $h$ is holomorphic in the interior of $C^{1}$, then for any $y$ in the interior of $C^{1}$, the function on $R$ :

$$
x \rightarrow h(x+y)
$$

defines a distribution on $R$; we denote this distribution by $\tau_{y} h$. A similar notation applies to the other octants.

Let $W$ be a space of functions or distributions ( $W$ not necessarily analytically uniform). Let $T \in W$ and suppose there exist functions $h^{j}$ with $h^{j}$ holomorphic in the interior of $C^{j}$ such that for each $j$ and each appropriate $y$ we can consider $\tau_{y} h^{j}$ as an element of $W$ in a natural way (such that the map $y \rightarrow \tau_{y} h^{j}$ is an analytic map of interior $C^{j}$ into $W$ ) and such that the limit

$$
\lim _{|y| \rightarrow 0} \tau_{y} h^{i}=T^{i}
$$

exists in the topology of $W$, and

$$
T=T^{1}+\cdots+T^{2^{n}} .
$$

Then we also write

$$
T=h^{1}+\cdots+h^{n}
$$

We give now a criterion for every $T \in W$ to have a representation of the form (43) : 
THEOREM 9. Let $W$ be analytically uniform and let $K$ be an analytical uniform structure of $W$. Suppose the functions $k$ of $K$ can be chosen to have the following properties:

$$
\exp (-t|z|)=O(k(z))
$$

for any $t>0$. Then every $T \in W$ can be written in the form (43).

Proof. By Theorem 1 we can write

$$
T=T^{1}+\cdots+T^{2^{n}}
$$

where

$$
T^{j}=\int \exp (i x \cdot z) d \mu^{j}(z) / k^{j}(z)
$$

where $k^{i} \in K$ and $\mu^{i}$ is a bounded measure with support $\mu^{j} \subset C^{j}$. For any $y$ in the interior of $C^{i}$ it is clear that the integral

$$
h^{j}(x+y)=\int \exp (i(x+y) \cdot z) d \mu^{j}(z) / k^{j}(z)
$$

converges absolutely and uniformly for $y$ in any compact set of the interior of $C^{j}$. Thus, $h$ is holomorphic in the interior of $C^{j}$. We claim that, for any such $y, \tau_{y} h^{i}$ defines, in a natural way an element of $W$. For any $F \in \hat{W}^{\prime}$, the integral

$$
\int F(z) \exp (i y \cdot z) d \mu^{i}(z) / k^{j}(z)
$$

is absolutely convergent; moreover, these integrals are uniformly bounded on the set of $F$ which satisfy $\left|F(z) / k^{j}(z)\right| \leqq 1$ for all $z \in C$, that is, these integrals are uniformly bounded for $F$ in a neighborhood of zero in $\hat{W}^{\prime}$. This means that the map

$$
f \rightarrow \int F(z) \exp (i y \cdot z) d \mu^{i}(z) / k^{i}(z)
$$

( $f \in W^{\prime}, F$ is the Fourier transform of $f$ ) defines a continuous linear function on $W^{\prime}$, which it is natural to call $\tau_{y} h^{j}$. It is readily verified that $y \rightarrow \tau_{y} h^{j}$ is an analytic map of interior of $C^{j}$ into $W$. Finally, a simple "abelian" theorem shows that as $|y| \rightarrow 0$,

$$
\tau_{y} h^{i} \rightarrow T^{i}=\int \exp (i x \cdot z) d \mu^{i}(z) / k^{j}(z)
$$

This, together with (44) and (45), completes the proof of Theorem 9.

REMARK. In case $W$ is one of the spaces of distributions of Schwartz, the identification of $\tau_{y} h^{j}$ with a distribution which we made in the proof of 
Theorem 9 is readily verified to be the same as Schwartz' way of making a distribution of the function on $R: x \rightarrow h^{j}(x+y)$.

Global approximation.

THEOREM 10. Let $n=1$, and let $f \in E$; then we can find an entire function $g$ such that $f-g$ is in the space $O_{M}^{\prime}$ of Schwartz. Again, let $n=1$ and let $T \in D_{r}^{\prime}$. Then we can find an entire function $h$ such that $T-h$ is in the space $S^{\prime}$ of Schwartz.

Proof. For the first part, we use Theorem 6. Then, we can find bounded measures $\mu_{1}, \mu_{2}$ with support $\mu_{1} \subset$ imaginary axis, support $\mu_{2} \subset$ real axis, and we can find continuous positive monotonic functions $l_{1}$ and $l_{2}$ on $R$ such that $l_{1}$ tends to infinity faster than any exponential and $l_{2}$ tends to infinity faster than any polynomial, and such that

$$
f(x)=\int \exp (i x \cdot z) d \mu_{1}(z) / l_{1}(z)+\int \exp (i x \cdot z) d \mu_{2}(z) / l_{2}(z)=g+g_{1}, \text { say }
$$

Clearly $g$ is entire and it is readily verified that $g_{1} \in O_{M}^{\prime}$.

The proof for $D_{F}^{\prime}$ is similar.

In Theorem 10, and in the corresponding Theorem 6, we have used the fact that the functions of $\hat{E}^{\prime}$ are entire functions of exponential type. Actually, we can define spaces $W$ which are analytically uniform but for which the functions $k \in K$ are entire functions with other kinds of growth conditions at infinity. Examples of such spaces are constructed in [2] where we get certain spaces of entire functions of finite order. The result corresponding to Theorems 5 and 6 for these spaces is that the lines $A_{j}, B_{j}$ in the complex $z_{j}$ plane should be replaced by a finite set of half-planes through the origin such that the sectors obtained are of sufficiently small angle.

For the approximation problem corresponding to Theorem 10 for these questions, the following abstract situation prevails. (For simplicity we assume $n=1$.) The set $K$ consists of all functions $k(z)=k_{1}(\xi) k_{2}(\eta)$ (where $z=\xi+i \eta)$ such that $k_{1}$ grows faster than any polynomial and $k_{2}$ tends monotonically to infinity faster than some set of functions; $k_{2}$ always tends to infinity faster than any exponential. The problem of approximation is then to find the largest (at infinity) function $\lambda$ such that the conditions

$$
\begin{aligned}
|F(\xi)| & \leqq k_{1}(\xi), \\
|F(\xi+i \eta)| & \leqq k_{1}(\xi) k_{2}(\eta) \quad \text { for } \eta \geqq \lambda(\xi)
\end{aligned}
$$

imply $|F(z)| \leqq k(z)$ for all $z$, for $F \in \hat{W}^{\prime}$, for a set of $k$ sufficient to define the topology of the space $\hat{W}^{\prime}$. For then, by our above method we derive for any $f \in W$

$$
f(x)=\int \exp (i x \xi) d \mu_{1}(\xi) / k_{1}(\xi)+\int \exp (i x \xi) d \mu_{2}(z) / k(z)=f_{1}+f_{2}, \text { say, }
$$


where $\mu_{1}$ and $\mu_{2}$ are bounded measures and $\mu_{2}$ has its support on $\eta \geqq \lambda(\xi)$. Since $k_{2}$ is in general larger than $k_{1}$, it is clear that the larger we can make $\lambda$, the more regular $f_{2}$ will be. In particular, if we can make $\lambda \rightarrow \infty$ faster than $\epsilon \xi$ for some $\epsilon>0$, then we see easily that $f_{2}$ will be entire. In case $W$ is the space of indefinitely differentiable functions $f$ such that $f$ and all its derivatives are $O\left(\exp \left(|x|^{a+c}\right)\right.$ for every $\left.\epsilon>0\right)$ (see [4]) we derive the result that

$$
f=f_{1}+f_{2},
$$

where $f_{1} \in O_{M}$ and $f_{2}$ is an entire function of order $\leqq a$.

Application to the heat equation. Let $t$ be a real variable and let us consider a function $g(t, x)$ which satisfies the heat equation

$$
\frac{\partial g}{\partial t}=\frac{\partial^{2} g}{\partial x_{1}^{2}}+\cdots+\frac{\partial^{2} g}{\partial x_{n}^{2}}
$$

for all $t, x$. It is well known that for fixed $t$ the function $x \rightarrow g(t, x)$ is entire. We can use our methods to prove

TheORem 11. Let $f(x) \in H$. Then there exists a $g$ which is a solution of the heat equation for all $t, x$ such that $g(0, x)=f(x)$.

Proof. We use Theorem 5 and we choose $A_{j}$ as the line $\arg z_{j}=\pi / 4$, $\arg z_{j}=5 \pi / 4$ and $B_{j}$ as the line $\arg z_{j}=3 \pi / 4$, arg $z_{j}=7 \pi / 4$. Then we may write

$$
f(x)=\int \exp (i x \cdot z) d \mu(z) / k(z)
$$

where $k(z)$ grows faster than any exponential and $\mu$ is a bounded measure with support on $\pi\left(A_{j} \cup B_{j}\right)$.

Define

$$
g(t, x)=\int \exp \left(i x \cdot z-t\left(z_{1}^{2}+\cdots+z_{n}^{2}\right)\right) d \mu(z) / k(z) .
$$

First we notice that for $z_{j} \in A_{j}$ or $z_{j} \in B_{j}$, we have $z_{j}^{2}$ pure imaginary, so that $\left|\exp \left(-t\left(z_{1}^{2}+\cdots+z_{n}^{2}\right)\right)\right|=1$. Thus the integral in (54) converges absolutely and uniformly together with its derivatives uniformly for $t, x$ in compact sets. Hence we can differentiate under the integral sign and deduce that $g$ satisfies the heat equation. It is clear that $g(0, x)=f(x)$ so our result is demonstrated.

REMARK. Theorem 11 is a special case of a general existence theorem for Cauchy's problem on a characteristic surface. In a separate article we show by similar but more complicated methods that for any linear partial differential equation with constant coefficients we can assign Cauchy data which are arbitrary entire functions on any hyperplane. This result is, of course, new 
only in case the hypersurface is characteristic; otherwise it is contained in the Cauchy-Kowalewski theorem.

Application to division problems. In [3, p. 584], we posed the following problem (Problem 4): Is $D * E=E$ ? We shall now show that the response is in the affirmative. For simplicity we consider first the case $n=1$.

Let $f \in E$; then by Theorem 6 we may write

$$
f=\boldsymbol{g}+\boldsymbol{h},
$$

where $h$ is entire and $g$ is of the.form

$$
g(x)=\int \exp (i x \cdot z) d \mu(z) / l(z)
$$

with $\mu$ a bounded measure whose support is on $R$ and $l$ tends to infinity faster than any polynomial. Suppose we can find a function $b \in D, s \in E$ such that

$$
b * s=g \text {. }
$$

Then we are reduced to finding a $t \in E$ satisfying

$$
b * t=h \text {. }
$$

But such a $t$ always exists by a theorem of Malgrange and of the author (see [2]). Thus we are reduced to the construction of $b$ and of $s$.

We shall construct $b$ in such a way that the Fourier transform $B$ of $b$ tends to zero on $R$ less rapidly than $[l(z)]^{-1 / 2}$, that is, $B$ will have the property that

$$
[l(x)]^{-1 / 2} B(x)^{-1} \rightarrow 0 \text { as } x \rightarrow \pm \infty .
$$

Thus, if we call $s$ the inverse Fourier transform of $\mu / l B$, then it is clear that $s \in E$ (even that $s \in O_{M}$ ) and we see immediately that

$$
b * s=g \text {. }
$$

It remains thus to construct $B \in \hat{D}$ with the desired property. Set

$$
v(z)=\sin (z+i) /(z+i) \sin i
$$

so $v$ is an entire function of exponential type with $|v(0)|=1$ and $v(x)$ behaves essentially like $(1+|x|)^{-1}$. Without going into the details, it is easy to see that we can construct $B$ of the form

$$
B(z)=\prod_{j=1}^{\infty} v(\epsilon, z)
$$

for suitable $\boldsymbol{\epsilon}_{j}$.

In case $n>1$, we again use Theorem 6 with the $B_{j}$ of that theorem being the imaginary axis of the complex $z_{j}$ plane. Then we proceed along lines similar to the above with two modifications: 
(a) We cannot use the result of Malgrange and the author, but we must make estimates along the imaginary axis also.

(b) We must replace the function $B$ used above by a product of functions $B\left(z_{1}\right) B\left(z_{2}\right) \cdots B\left(z_{n}\right)$. Here the function $l$ gets replaced by

$$
l_{2}(x)=\min l_{2}^{j}(x)
$$

and on the imaginary axis we use

$$
l_{1}(x)=\min l_{1}^{j}(x)
$$

We have thus

Theorem 12. $D * E=E$.

We wish now to give an application to division problems depending on a parameter (see [3, Problem 8, p. 585]). In [3] we introduced the concept of slowly decreasing. A function $J \in \hat{E}^{\prime}$ is called slowly decreasing if there is an $a>0$ such that for any $x \in R$ there is a $y \in R$ with $|x-y|<a \log (1+|x|)$ and $|J(y)| \geqq(a+|x|)^{-a}$. It is shown in [3] that for $S \in E^{\prime}$ the existence of a fundamental solution for $S$ is equivalent to having the Fourier transform of $S$ slowly decreasing. By using the minimum modulus theorem in the form used in [3] we can show

Lemma 13. $J$ is slowly decreasing if and only if we can find $a b>0$ so that the set

$$
B_{b}(J)=\left\{z|| J(z) \mid \geqq(b+|z|)^{-b} \exp (-b|I z|)\right\}
$$

is sufficient to define the topology of $\hat{E}^{\prime}$.

The statement that $B_{b}$ is sufficient to define the topology of $\hat{E}^{\prime}$ means that, if $K=\{k\}$ is an analytic structure for $\hat{E}^{\prime}$ then the topology of $\hat{E}^{\prime}$ can be defined by the semi-norms

$$
\sup _{z \in B_{b}(J)}|F(z)| / k(z)
$$

for $F \in \hat{E}^{\prime}$. As in the cases treated above this means that also any $f \in E$ is of the form (1) where support $u \subset B_{b}(J)$.

Let $\left\{J_{\lambda}\right\}_{\lambda \in \Delta}$ be a family of functions in $\hat{E}^{\prime}$ each of which is slowly decreasing. We say that $\left\{J_{\lambda}\right\}$ is uniformly slowly decreasing if there is a $b>0$ so that the set

$$
B_{b}\left(\left\{J_{\lambda}\right\}\right)=\bigcap_{\lambda \in \Delta} B_{b}\left(J_{\lambda}\right)
$$

is sufficient to define the topology of $\hat{E}^{\prime}$.

Next, let $\Lambda$ be a locally compact space. Let $\lambda \rightarrow S_{\lambda}$ be a continuous map 
of $\Lambda$ into $E^{\prime}$ and let $\lambda \rightarrow T_{\lambda}$ be a continuous map of $\Lambda$ into $D^{\prime}$. We ask whether there exists a continuous map of $\lambda \rightarrow V_{\lambda}$ of $\Lambda$ into $D^{\prime}$ such that

$$
S_{\lambda} * V_{\lambda}=T_{\lambda}
$$

THEOREM 14. Suppose that $S_{\lambda}$ is slowly decreasing for each $\lambda$ and that $\left\{S_{\lambda}\right\}_{\lambda \in L}$ is uniformly slowly decreasing whenever $L$ is compact. Then there exists a continuous map $\lambda \rightarrow V_{\lambda}$ of $\Lambda$ into $D_{1}$ such that

$$
S_{\lambda} * V_{\lambda}=T_{\lambda}
$$

Proof. By taking a partition of unity in $\Lambda$ we may easily reduce the problem to the case $\Lambda$ compact which we now assume; let $B$ be the set $B_{b}(\{\Lambda\})$ whose existence is guaranteed by the fact that the set $\left\{J_{\lambda}\right\}$ of Fourier transform is uniformly slowly decreasing.

For each $\lambda$, call $U_{\lambda}$ the Fourier transform of $T_{\lambda}$, that is, $U_{\lambda} \cdot F=T_{\lambda} \cdot f$ for $f \in D, F \in D$ being the Fourier transform of $f$. Then we may consider the map $\lambda \rightarrow U_{\lambda}$ as an element of $L(D, N(\Lambda))$, the space of continuous linear maps of $\hat{D}$ into $N(\Lambda)$, the space of continuous functions on $\Lambda$. Call $M$ the space of continuous functions $G$ on $B$ such that for any $k \in K$ we have $|G(z)| / k(z) \rightarrow 0$ for $z \in B,|z| \rightarrow \infty$ with the topology defined by the semi-norms

$$
\sup _{z \in B}|G(z)| / k(z) \quad \text { for } z \in K .
$$

We now use the fact that $\hat{D}^{\prime}$ is nuclear (see A. Grothendieck, Produits tensoriels topologiques et espaces nucleaires, Memoirs Amer. Math. Soc. no. 16, 1955). From the results of Grothendieck it follows that each $U_{\lambda}$ can be extended to an element $\tilde{U}_{\lambda} \in M^{\prime}$ in such a way that $\lambda \rightarrow \tilde{U}_{\lambda}$ is continuous. We now define $V_{\lambda}$ as the inverse Fourier transform of $J_{\lambda}^{-1} \sigma_{\lambda}$, that is,

$$
V_{\lambda} \cdot f=\tilde{U}_{\lambda} \cdot J_{\lambda}^{-1} F,
$$

where $f \in D, F$ is the Fourier transform of $f$. It is easily verified that $V_{\lambda} \in D^{\prime}$, that

$$
S_{\lambda} * V_{\lambda}=T_{\lambda}
$$

and that $\lambda \rightarrow V_{\lambda}$ is continuous from $\Lambda$ into $D^{\prime}$. This completes the proof of Theorem 13.

4. General remarks. 1. In connection with Theorem 1 , it is of interest to know that, in many cases, one could show directly the existence of the Fourier representation (1). We illustrate for the case $n=1$, the space $E$ : We take a partition of unity on $R$ relative to the sets $[j-1 \leqq x \leqq j+1]$. Then for any $f \in E$ we write $f=\sum f^{i}$ where support $f^{i} \subset[j-1 \leqq x \leqq j+1]$. Each $f^{i}$ has a Fourier transform $F^{i}(z)$, and for $j>1$ for example, we have

$$
f^{i}(x)=\int_{R} F^{i}(z) \exp (-i x z) d z=\int_{R+i y_{j}} F^{i}(z) \exp (-i x z) d z .
$$


Now for $y_{j}$ large positive, $F^{j}(z)$ is very small on $I z=y_{j}$; in fact $F^{j}(z)$ behaves essentially like $\exp \left(-(j-1) y_{j}\right)$. By choosing the $y_{j}$ properly, we can define $\mu(z) / k(z)$ as the sum of the measures $F^{i}(z)$ times the measure $d z$ on the line $I z=y_{j}$. The precise construction of the $y_{j}$ can be carried out by the method of [1] used to prove the description of the topology of $D_{F}^{\prime}$. The possibility of such a direct construction was pointed out to me by Professor Mikusinski whom I should like to thank. A similar method, except that the sets $R+i y_{j}$ are replaced by suitable polycylinders, can be used to prove the analogous result for $H$. (This was pointed out to me by Hörmander.) However, the above method does not seem to yield the more refined results such as those in $\$ 2$ which are needed for the applications.

2. We can give also a simple proof of the representation of a continuous function as a sum $f=h^{1}+\cdots+h^{2^{n}}$ where $h^{i}$ is holomorphic in the interior of $C^{i}$. By a result of Poincaré (see e.g. [4]) we can find an entire function $g$ with no zeros so that $f(x) / g(x) \rightarrow 0$ exponentially as $|x| \rightarrow \infty$. Then if we call $m(x)=f(x) / g(x)$, the Cauchy integral formula allows us to write $m=m^{1}+\ldots$ $+m^{2^{n}}$ where $m^{i}$ is holomorphic in the interior of $C^{j}$. On writing

$$
f=g m=g m^{1}+\cdots+g m^{2^{n}}
$$

we have our desired result.

However, this method does not give such strong results as those obtained in Theorem 9, for this method does not work for distributions; also if $f \in E$ then this direct method does not show that $\tau_{y} h^{i}$ converges in the topology of $E$ as $|y| \rightarrow 0, y \in$ interior $C^{j}$. To prove the analogous result for $E$, we should have to prove that there exists an entire function $g$ such that $g$ and all its derivatives are arbitrarily small at infinity. We do not know how to construct such a function.

A second direct method to obtain a representation as in Theorem 9 would be to take a partition of unity as in Remark 1 above. Then we write (say $n=1) f=\sum f^{i}$, where support $f^{j} \subset[j-1 \leqq x \leqq j+1]$. We can write each $f^{j}$ as the sum $p_{1}^{j}+p_{2}^{j}$ where $p_{1}^{j}$ is holomorphic in the upper half-plane and $p_{2}^{j}$ is holomorphic in the lower half-plane. The $p_{k}^{s}$ could be constructed by the usual Cauchy integral formula, but then the series $\sum_{j} p_{k}^{j}(z)$ would not converge. We must instead use Cauchy's integral formula in the form

$$
u(z)=\left(\exp \left(i y_{j} \cdot z\right) / 2 \pi i\right) \int \exp \left(-i y_{j} \cdot \omega\right) u(\omega) \frac{d \omega}{\omega-z}
$$

for suitable $y_{j}$, where the path of integration is, as usual, a simple, rectifiable, closed Jordan curve containing $z$ in its interior. This method is closely related to the method of Remark 1 above, and corresponds to breaking up the integrals used there which were on $R+i y_{j}$ into integrals on $R^{+}+i y_{j}$ and $R^{-}+i y_{j}$ where $R^{ \pm}$is the positive (negative) real axis. 
3. In our definition of analytically uniform, we have used a description of $W^{\prime}$ by uniform norms. It should also be of interest to consider other types of norms, for example, types of integral norms.

4. A very important, and doubtless very difficult, problem is to be able to construct minimal sets for the support of $\mu$ in our representation (1).

5. Finally the problem arises of understanding directly (without Fourier transform) the concept of an analytically uniform space. A true understanding of this concept might provide an insight into the treatment of variable coefficient partial differential equations.

\section{BibLIOGRAPHY}

1. L. Ehrenpreis, Solutions of some problems of division, Part I, Amer. J. Math. vol. 76 (1954) pp. 883-903.

2. , Solutions of some problems of division, Part III, Amer. J. Math. vol. 78 (1956) pp. 685-715.

3. - Solutions of some problems of division, Part IV, Amer. J. Math. vol. 82 (1960) pp. 522-587.

4. - Theory of infinite derivatives, Amer. J. Math. vol. 81 (1959) pp. 799-845.

5. - The fundamental principle and some of its applications, Proceedings of the Symposium on Linear Spaces, Jerusalem, 1960.

6. L. J. Schwartz, ThEorie des distributions, vols. I, II, Paris, Hermann \& Cie, 1950, 1951.

YESHIVA UNIVERSITY,

New YoRK, New YoRK 